\title{
A Closer Insight into The Role of Vitamin D in Polycystic Ovary Syndrome (Pcos)
}

\author{
Alshaymaa Amin Zaki El-Bahya1*, Rasha Ali Radwanb², Mohamed Zakaria Gadc ${ }^{3}$ and Sahar Mohamed Abdel \\ Maksoudc ${ }^{3}$
}

${ }^{1}$ M. Pharm Research Scholar, Monad University, India

Submission: October 19, 2018; Published: November 19, 2018

*Corresponding author: Alshaymaa Amin Zaki El-Bahy, Ph. D, Faculty of Pharmacy, Heliopolis University, Misr-Ismailia Desert Road, Kilo 20, P.O. Box: 2834, El Horreya Heliopolis, Cairo, Egypt

\begin{abstract}
Polycystic ovary syndrome (PCOS) is an endocrine disorder characterized by anovulation, menstrual disorder, amenorrhea, hirsutism, and infertility. According to the Rotterdam consensus workshop to diagnose PCOS, at least two of the cardinal symptoms of PCOS must be fulfilled. Women with PCOS appear to have a higher risk of developing metabolic and cardiovascular disorders. Recent attention is being directed towards the role of vitamin D in several diseases such as cancer, neurodegenerative and autoimmunine disorders. The present review introduces the role of vitamin D, its mechanism of action in the management of PCOS. In addition to summarizing the pathophysiology, clinical manifestations, diagnosis and treatment of PCOS from previous published data.
\end{abstract}

Keywords: polycystic ovary syndrome; hyperandrogenism; metabolic disorders; vitamin D

\section{Introduction to the Polycystic Ovary Syndrome}

Infertility is a complex disorder with significant medical, psychosocial, and economic aspects [1], affecting about $15 \%$ of couples [2]. One major cause of female infertility is polycystic ovary syndrome (PCOS). PCOS is an endocrine disorder characterized by anovulation, menstrual disorder, amenorrhea, hirsutism, and infertility. Women with PCOS have impaired metabolism of androgen and estrogen and they are often obese, insulin resistant, and at risk for type 2 diabetes and cardiovascular disease [3]. PCOS has two definitions, set in1990 by the National Institutes for Health (NIH) and in 2003

Table 1: PCOS phenotypes according to the Rotterdam consensus.

\begin{tabular}{|c|c|c|c|c|}
\hline & Severe PCOS & Mild PCOS & Ovulatory PCOS & Hyperandrogenism and Chronic Anovulation \\
\hline Periods & Irregular & Irregular & Normal & Irregular \\
\hline $\begin{array}{c}\text { Ovaries on } \\
\text { Ultrasonography }\end{array}$ & Polycystic & Polycystic & Polycystic & High \\
\hline Androgen concentration & High & Mildly raised & High & Increased \\
\hline Insulin concentrations & Increased & Normal & Increased & Potential long term \\
\hline Risks & Potential long term & Unknown & Unknown & \\
\hline
\end{tabular}

\section{Pathogenesis}

The exact etiology of PCOS has not yet been determined, however there are many suggested hypotheses including

\section{Androgen Abnormalities}

$60-80 \%$ of women with PCOS have high circulating testosterone [4,5], while $25 \%$ of them have high levels of by the Rotterdam consensus workshop. The NIH requires the signs of hyperandrogenism. The Rotterdam consensus requires the presence of two or all of three features: clinical and/or biochemical hyperandrogeneism, chronic anovulation and polycystic ovaries Rotterdam (2004). The Rotterdam criteria added ovarian morphology, expanding the inclusion criteria, and thus increasing the prevalence of PCOS Dewailly, CatteauJonard et al. (2006). As the 2003 Rotterdam consensus included ovarian morphology in PCOS diagnosis, four phenotypes of PCOS are recognized (Table 1). presence of chronic anovulation and clinical or biochemical

dehydroepiandrosterone [6]. Follicles in PCOS have thickened thecal layers, which show excessive steroidogenic enzyme expression and activity. This excessive activity is attributed to either genetic factors [7] or impaired intracellular signaling [8]. Chronic excessive secretion of androgen from ovaries and adrenals results in abdominal adiposity and android obesity in affected women [9]. Abdominal adiposity leads to 
hypoadiponectinemia and adipose tissue dysfunction [10]. Hypoadiponectemia is associated with insulin resistance, type 2 diabetes, metabolic syndrome, and obesity-related cardiovascular disease Matsuzawa (2005).

\section{Hypothalamic-Pituitary Axis Abnormality}

Luteinizing hormone (LH) hypersecretion is a characteristic hallmark of PCOS sufferers. The balance between LH and follicle stimulating hormone (FSH) is impaired in women with PCOS, as LH secretion is increased, and FSH levels are decreased leading to an abnormal ratio of circulating LH to FSH around 2:1 DeVane, Czekala et al. (1975). LH is secreted in a pulsatile manner. Women with PCOS have an increase in both the LH pulse frequency and amplitude. This increase in LH secretion is thought to be a consequence of increased frequency of hypothalamic gonadotropin-releasing hormone (GnRH) pulses. Increased LH, in turn, leads to an increase in androgen production by the theca cells within the ovary [10].

\section{Insulin Action Abnormalities}

Insulin resistance (IR) is the decreased insulin-mediated glucose utilization. Up to $50 \%$ of obese and non-obese women with PCOS suffer from hyperinsulinemia and higher degrees of IR than healthy women of the same age and body mass index (BMI) Dunaif, Segal et al. (1989). However, these numbers may vary depending on the sensitivity and specificity of the tests used for assessment. Hyperinsulinemia may lead to hyperandrogenism, since high serum insulin enhances LH-induced androgen production by theca cells in the ovaries Bergh, Carlsson et al. (1993). In addition, insulin inhibits Sex hormone-binding globulin (SHBG) production by the liver, increasing bioavailable testosterone Nestler, Powers et al. (1991). Excess of intraovarian androgen leads to anovulation, as excess androgens causes premature luteinization of follicles [11].

\section{Abnormal Folliculogenesis}

The polycystic ovaries have two to six times more primary, secondary and antral follicles than normal ovaries [12]. The exact reason for these high numbers is unknown. However, it is suggested to occur due to increased androgen secretion. A positive correlation was established between circulating androgen levels and number of follicles Pache, de Jong et al. (1993). Moreover, deficient growth signals from oocytes may lead to abnormal folliculogenesis (Jonard and Dewailly 2004).

\section{Clinical Features and Diagnosis of PCOS}

a. To confidently diagnose PCOS, diseases having similar clinical presentation should be excluded such as; congenital adrenal hyperplasia, Cushing's syndrome, androgen-secreting tumors, thyroid dysfunction, hypogonadotropic hypogonadism and premature ovarian failure [13].

b. The cardinal features to diagnose PCOS are hyperandrogenism, anovulation and polycystic ovaries. Moreover, in adults, ovaries are best examined using transvaginal ultrasonography [14]. The optimal time to examine ovaries is during the follicular phase. Ovary with more than 12 follicles measuring from 2-9 $\mathrm{mm}$ in diameter and ovarian volume $>10$ $\mathrm{ml}$ are enough criteria to make a diagnosis of polycystic ovaries (Figure 1).

c. Other clinical features of PCOS are obesity, mainly central obesity, affecting more than $50 \%$ of women with PCOS Gambineri, Pelusi et al. (2002), hirsutism and acanthosis nigricans. Acanthosis nigricans, is the dermal manifestation of insulin resistance. It consists of mucocutaneous eruptions, which mainly occur in the axillae, nape and skin flexures, and characterized by hyper-pigmentation Schwartz (1994). It affects $1-3 \%$ of PCOS patients [4], and is more common in adolescents Sampaolo, Livieri et al. (1994).

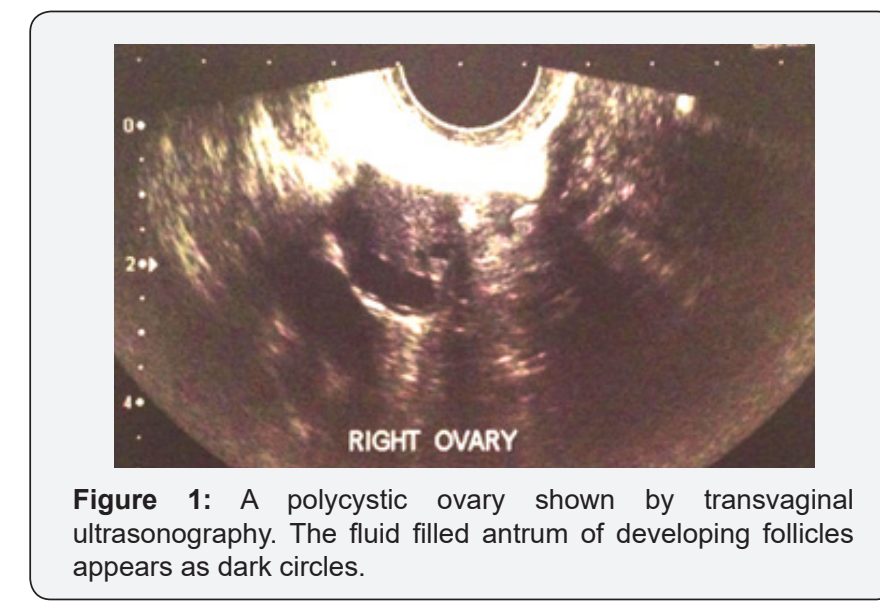

\section{Complications of PCOS and its Impacts}

\section{Health Impact}

Reproduction and metabolic disorders in a recent study rates of hospital admission for women with PCOS were compared vs. age matched non-PCOS. It was found that women with PCOS were at higher rates of infertility ( 40.9 vs. $4.6 \%$ ), and miscarriages (11.1 vs. 6.1\%) and more likely to seek IVF (17.2 vs. 2.0\%) [15]. Prolonged anovulation leads to the continuous stimulation of the endometrium by estrogen, with no concomitant production of progesterone, putting women at risk of endometrial cancer due to endometrial hyperplasia Hardiman, Pillay et al. (2003). Women with PCOS are at high risk of type 2 diabetes mellitus (T2DM), no matter lean or obese; because of IR Dunaif, Segal et al. (1992).

PCOS also predisposes to metabolic syndrome. The criteria of metabolic syndrome are shown in (Table 2). Three out of the five criteria qualify for the syndrome Rotterdam (2004). PCOS is also associated with sleep apnea Tasali, Van Cauter et al. (2006), and possibly to increased risk of cardiovascular disease due to dyslipidemia, T2DM, and metabolic syndrome. In addition to endothelial vascular dysfunction, many inflammatory and thrombotic biochemical markers of CVD are present in excess in PCOS. However still more evidence is needed to determine whether these values are of real predictive power [16]. PCOS is also associated with non-alcoholic fatty liver disease [17]. 
Table 2: Criteria for metabolic syndrome in women with PCOS.

\begin{tabular}{|c|c|}
\hline Risk factor & Cutoff \\
\hline $\begin{array}{c}\text { Abdominal obesity (waist } \\
\text { circumference) }\end{array}$ & $>88 \mathrm{~cm}$ \\
\hline Triglycerides & $\geq 150 \mathrm{mg} / \mathrm{dl}$ \\
\hline HDL-C & $<50 \mathrm{mg} / \mathrm{dl}$ \\
\hline Blood pressure & $\geq 130 / \geq 85$ \\
\hline $\begin{array}{c}\text { Fasting and 2-h glucose from oral } \\
\text { glucose tolerance test }\end{array}$ & $\begin{array}{c}110-126 \mathrm{mg} / \mathrm{dl} \mathrm{and} / \mathrm{or} 2 \mathrm{~h} \\
\text { glucose } 140-199 \mathrm{mg} / \mathrm{dl}\end{array}$ \\
\hline
\end{tabular}

\section{Psychological Impact}

PCOS patients are at higher risk of depression and anxiety disorder. Significantly larger number of PCOS patients than controls reported worry, difficulty in sleeping and phobias $[18,19]$. Depression has been associated with increased cortisol levels, increased sympathetic activity and decreased serotonin levels in the central nervous system, such features are associated with insulin resistance [20]. Farideh et al. reported the relationship between insulin resistance and psychiatric distress in PCOS (Reference).

In addition, several studies demonstrated a correlation between psychological distress scores in PCOS patients and their serum levels of androgen Zangeneh, Jafarabadi et al. (2012). It has been suggested that women with PCOS have a lower self-esteem, a more negative self-image, higher tendency to depression and psychological distress. This can be attributed to the masculine physical appearance of PCO sufferers; hyperandrogenism, obesity [21], hirsutism, cystic acne, seborrhea and hair loss [22]. The relationships between psychological health aspects and the clinical characteristics of PCOS are yet to be explained.

\section{Conventional Therapy for PCOS}

Two main aspects should be taken into consideration while treating PCOs; Normalization of menstrual cycles and treatment of clinical features. Normal ovulation and menstruation could be attained by modest weight loss with diet and exercise Legro, Barnhart et al. (2007). In addition to medical and surgical interventions. In PCOS, anovulation is a consequence of low follicle-stimulating hormone concentrations and the arrest of antral follicle growth in the final stages of maturation. Medications such as clomiphene citrate, tamoxifen, aromatase inhibitors, metformin, glucocorticoids, or gonadotropins or surgically by laparoscopic ovarian drilling are commonly used [23].

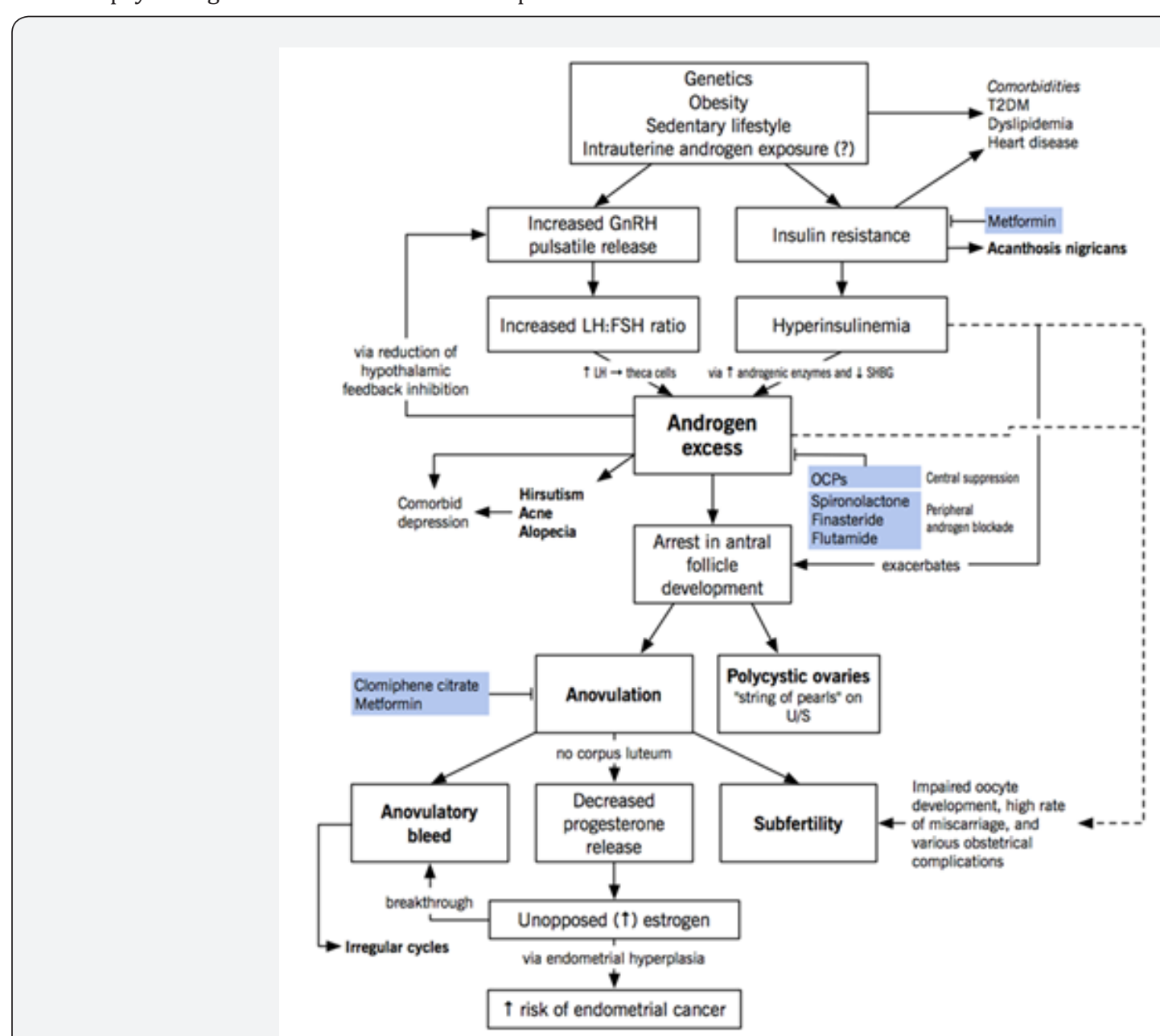

Figure 2: Summary of the clinical manifestation of PCOS and its conventional treatment. 


\section{Hormonal Therapy}

Oral agents such as Clomiphene citrate - a selective estrogen receptor modulator - is the first line of oral ovulationinduction agents used. Centrally, it blocks estrogen negative feedback on hypothalamus, leading to high levels of FSH and follicular growth and ovulation. Clomiphene citrate shows live birth rates near those of normal fertility Legro, Barnhart et al. (2007). In case of failure of these treatments, more advanced second line treatments are implemented such as injections of gonadotropins [24]. In addition, Laparascopic ovarian drilling, a minimal invasive technique, where multiple holes in ovarian stroma using laser are drilled. This results in a decrease in androgen production and allowing normal steroidogenesis and follicular development [24]. Intrauterine insemination and in vitro fertilization (IVF) are widely used assisted reproductive techniques to treat infertility in PCOS. Hormonal contraceptives whether estrogen only, progesterone only or both together are the first line treatment for menstrual irregularities, hirsutism and acne Legro, Arslanian et al. (2013). Anti-androgens such as spironolactone could be used to treat hirsutism. They are used in combination with hormonal contraceptives to ensure the additive effects of androgen suppression by hormonal contraceptives and androgen-blockade by anti-androgen (Figure 2).

\section{Drugs Improving Insulin Resistance}

Metformin- an insulin sensitizer- is a biguanide used in the management of type 2 diabetes mellitus. The benefits of metformin could be summarized as; improved ovulation, reduction in androgen levels and menstrual cycle regulation Sam (2003). and possible weight loss. Its mechanism of action could be explained as follows; inhibiting hepatic glucose production, decreasing intestinal glucose uptake and increasing insulin sensitivity in peripheral tissue Grundy (2002).

Moreover, Metformin improves ovulation via reducing insulin levels and altering the effect of insulin on ovarian androgen biosynthesis, theca cell proliferation, and endometrial growth. In addition to inhibiting ovarian gluconeogenesis with the subsequent reduction ovarian androgen production Badawy and Elnashar (2011). Troglitazone is another insulin-sensitizing drug that has been shown to improve ovulation and increase pregnancy rates with hepatotoxic side effects Azziz et al. (2011). Rosiglitazone and pioglitazone have been shown to enhance ovulation in women with PCOS. However, further studies are required.

\section{Life Style Modifications}

Obesity is observed in $35 \%-60 \%$ of women with PCOS [11]. Hyperandrogenism causes central obesity with a high waist/ hip ratio independent of the body mass index (BMI). Commonly, obesity is associated with anovulation, miscarriage, or late pregnancy complications (such as pre-eclampsia and gestational diabetes) Pasquali et al. (2003) and Boomsma et al. (2006). Weight loss improves the endocrine profile and increases the likelihood of ovulation and pregnancy. Normalization of the menstrual cycles and ovulation could occur with modest weight loss as little as $5 \%$ of the initial weight. Patel and, Nestler (2006). Diet and exercise are recommended for obese PCOS patients. It has been reported that low-fat diets produce a decrease in hyperinsulinemia, which improves metabolic effects.14 An increase in physical activity is recommended for PCOs 16 where several established the role of exercise in the treatment of obese PCOS patient Reaven, (2005).

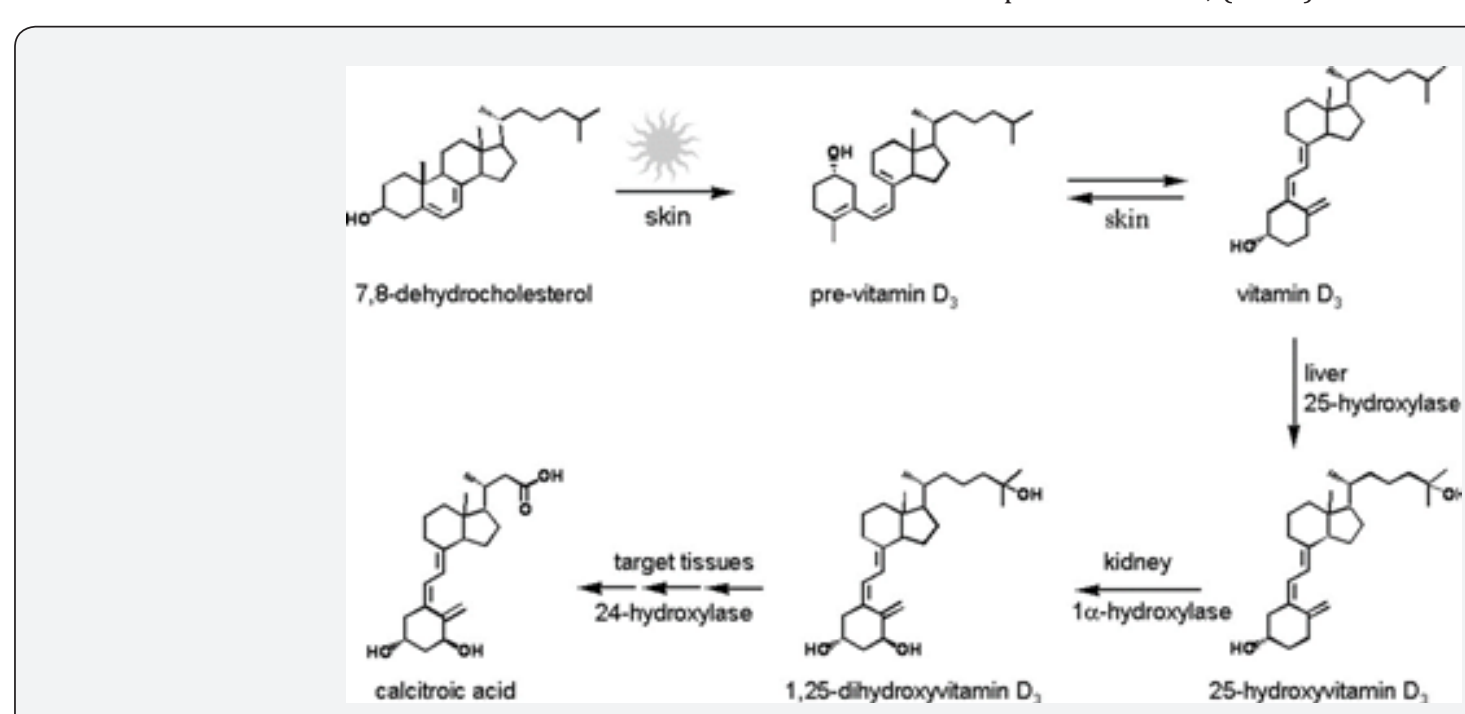

Figure 3: The synthesis of vitamin D3 and D2.

\section{Introduction to Vitamin D}

Vitamin D is a fat-soluble vitamin well known for its essential role in bone health [20]. It is converted in the body into several biologically active metabolites and interacts with the genome to produce both calcemic and nonracemic effects. Several studies have been proven that vitamin D influences several body functions including cellular proliferation, differentiation, immune function, vascular tone, insulin secretion, the renin angiotensin aldosterone system, and fertility. It has been proposed that hypovitaminosis D is linked to an increased risk 
for cancer Chandra (2011), autoimmune diseases, diabetes, and cardiovascular diseases [20] and PCOs.

\section{Synthesis of Vitamin D}

The major source of vitamin D is its endogenous cutaneous synthesis [25]. Endogenous vitamin D3 synthesis starts in the skin by the photolytic conversion of 7-dehydrocholesterol present in dermal fibroblasts and epidermal keratinocytes to provitamin D3 by the ultraviolet B (UVB) component of sunlight (290-315nm). Provitamin D3 isomerizes to vitamin D3 (Cholecalciferol) by body heat. It is then subjected to two hydroxylation reactions in liver and kidney to yield the biologically active form of vitamin D3; 1, 25-dihyroxy vitamin D3. Although the predominant source of vitamin D in humans is the endogenous vitamin D3, it could also be obtained from dietary sources. The dietary sources of vitamin D3 are fatty fish and egg yolk, vitamin D2 (ergocalciferol) is present in fungi and yeast which have been subjected to UVB light [20] (Figure 3).

\section{Physiological actions of Vitamin D3}

Biological effects of the active form of vitamin D3 (1,25-dihydroxyvitamin D3 [1,25(OH)2D3] or calcitriol) are mediated through a specific nuclear vitamin D receptor (VDR). Normal cells including the skeleton, parathyroid gland and reproductive organs as well as cancer cells express VDR $[20,26]$. This receptor belongs to the superfamily of nuclear receptors, which include receptors for steroids and thyroid hormones [12]. Once calcitriol binds to VDR, a conformational change of the receptor takes place which activates it. it dimerizes with retinoid X receptor RXR. The complex 1,25(OH)2D3-VDR-RXR then binds up or down to vitamin D-responsive elements (VDREs) regulating the transcription of various genes in the target cell [4]. This is the genomic action of vitamin D as shown in (Figure 4).

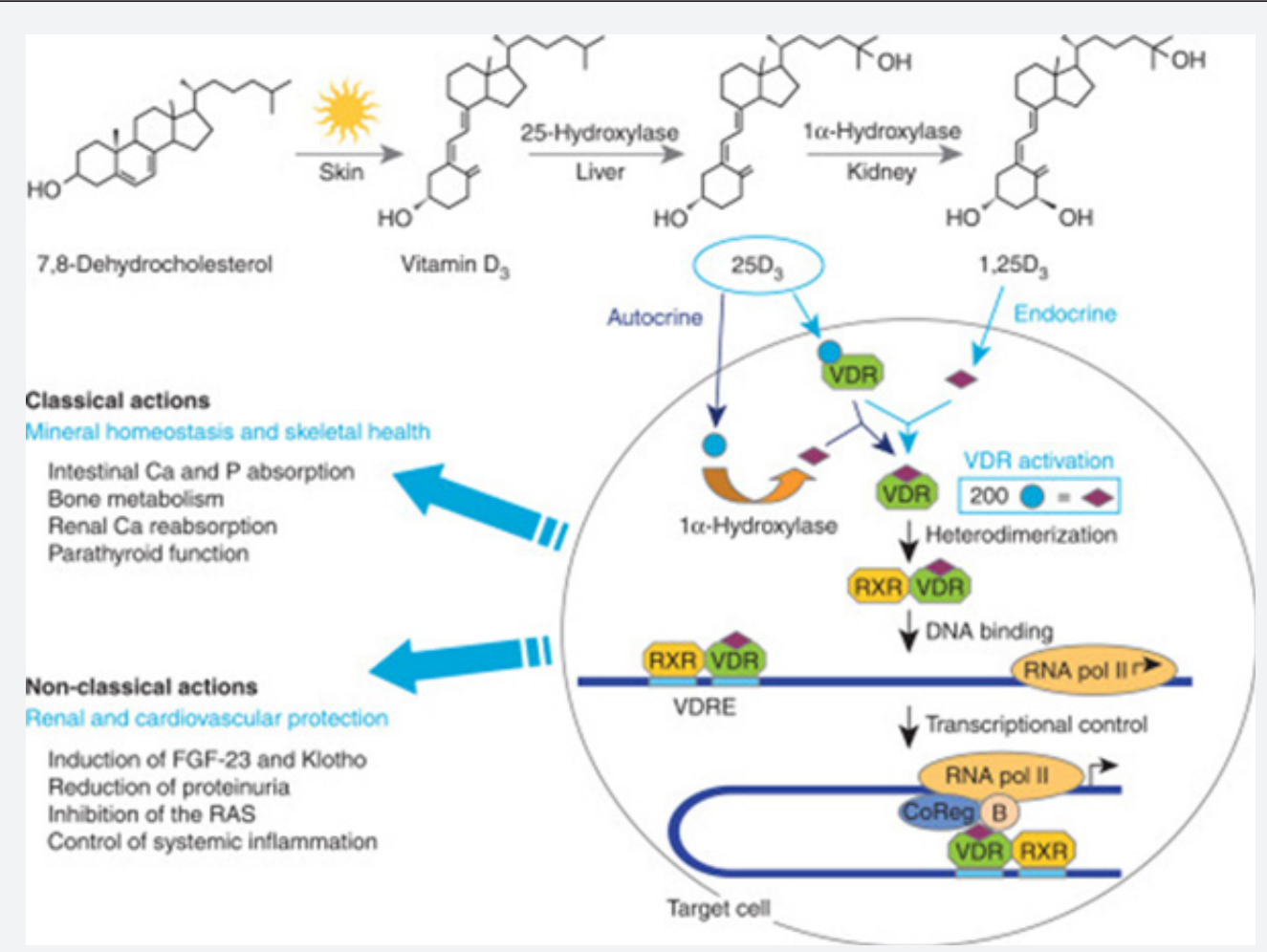

Figure 4: |Health benefits of VDR activation through genomic level. B, basal transcription factor; Ca, calcium; CoReg, coregulator molecule; FGF-23, fibroblast growth factor-23; P, phosphorus; RAS, renin-angiotensin system; RXR, retinoid $x$ receptor; VDRE, vitamin D-responsive element; 25D3, 25-hydroxyvitamin D3; 1,25D3, 1,25-dihydroxyvitamin D3.

Health benefits of VDR activation through genomic level. $\mathrm{B}$, basal transcription factor; Ca, calcium; CoReg, coregulator molecule; FGF-23, fibroblast growth factor-23; P, phosphorus; RAS, renin-angiotensin system; RXR, retinoid $x$ receptor; VDRE, vitamin D-responsive element; 25D3, 25-hydroxyvitamin D3; 1,25D3, 1,25-dihydroxyvitamin D3 [16]. VDR is thought to be involved in several health conditions and physiological functions whether at the genomic or non- genomic level. For the genomic level, it was found that more than 2700 human genome sites function through VDR binding [27]. Moreover, the 1,25(OH)2D can affect the expression of 229 genes, and at least 37 cell types that express VDR [28,29].

Examples of the genomic functions are the regulation of the calcium and phosphate transporters and their associated energy-driven ion-pumps in intestine and kidney [30-32], and the stimulation of bone resorption [33]. Some of the other noncalcemic actions include the regulation of genes involved in the differentiation of keratinocytes in the skin [22] and the functions of key cell types involved in innate and adaptive immunity, such as dendritic cells and T-cells [6]. 1,25(OH)2D3 has non-genomic 
actions, which are faster than the actions supported by genomic response. These actions may be mediated by cellular plasma membrane-binding VDRs. They may also involve a non-classical novel membrane called 1,25D3-MARRS (membrane-associated, rapid response steroid-binding $[7,8]$.

This may result in the activation of one or more second messenger systems, including phospholipase $\mathrm{C}$ (PKC), protein kinase C, G protein-coupled receptors, or phosphatidylinositol3-kinase (PI3K). This may result in either opening of the voltage- gated calcium or chloride channels or generation of the second messengers. Some of these second messengers, particularly RAF/MAPK, may modulate a cross-talk with the nucleus for gene expression regulation, hence there is a crosslink between Genomic and non- genomic actions [34,35] as shown in (Figure 5 ). Some of these non-genomic functions are the release of insulin from $\beta$-cells of pancreas, induction of calcium absorption from intestinal lumen and calcium influx into the muscle cells [25].

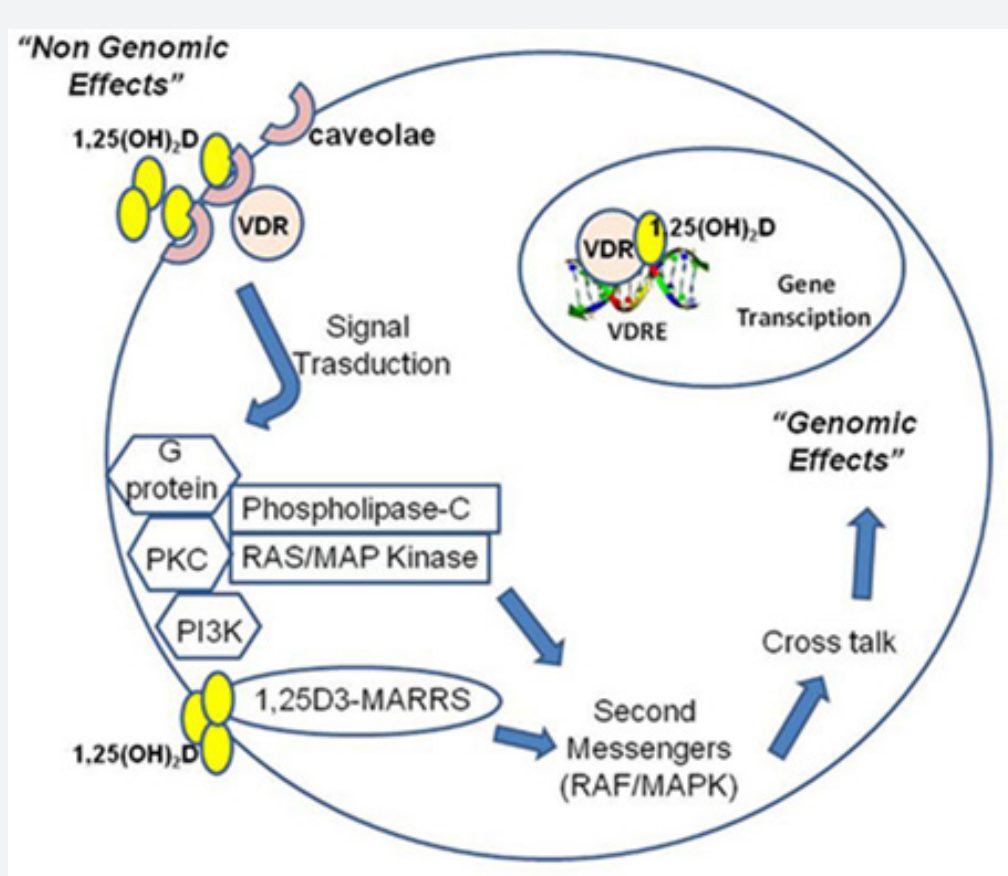

Figure 5: 1,25(OH)2D operates through nuclear receptor-mediated and plasma membrane-initiated mechanisms. 1,25(OH)2D interacts with the vitamin D receptor (VDR) localized in the cell nucleus to generate genomic effects or in caveolae of the plasma membrane to generate non-genomic effects.

\section{Correlation between Vitamin D Deficiency and PCO Features}

Several mechanisms are proposed to explain the role of Vitamin D deficiency in PCOS. First, in human ovarian tissue, calcitriol stimulates progesterone production by $13 \%$, estradiol production by $9 \%$, and estrone production by $21 \%$ [18]. Moreover, it has been proven that Vitamin D regulates oestrogen biosynthesis through direct regulation of the expression of the aromatase gene and by maintaining extracellular calcium homoeostasis. [21]. Hollaron [36], 1980 demonstrated that Vitamin D deficient mice have reduced fertility rates. Furthermore, VDR null mice demonstrated decreased aromatase activity in the ovary and impaired folliculogenesis $[21,36]$.

The augmentation of aromatase activity by vitamin D resulted in stimulation of oestrogen and progesterone production and lack of testosterone, in human ovarian tissue as proved by [18]. Second, it regulates human chorionic gonadotropin expression [37], and increases placental sex hormones production. Third, it has been demonstrated that calcitriol promotes calcium transport in the placenta [38], stimulates placenta lactogen expression [11] and regulates HOXA10 expression in human endometrial strom cells [1].

HOXA10 expression is important for the development of the uterus and essential for endometrial development, allowing uterine receptivity to implantation [26]. Finally, vitamin D deficiency increases PTH production. PTH is regulated through levels of serum calcium and vitamin D, and increased PTH is independently associated with PCOS, anovulatory infertility and increased testosterone [5]. In addition, Vitamin D deficiency is associated with calcium dysregulation, which contributes to the development of follicular arrest in women with PCOS and results in menstrual and fertility dysfunction. Moreover, for 2-3 months, thirteen vitamin D deficient women with PCOS (mean 250HD value was $11.2 \mathrm{ng} / \mathrm{ml}$ ) were supplemented with vitamin $\mathrm{D}$ combined with calcium to achieve normal ranges of $250 \mathrm{HD}$ levels (30-40 ng/ml) [39]. The results of this study were normalization of menstrual cycles within 2 months for seven of the nine women with menstrual dysfunction, two women became 
pregnant and the other four maintained their normal menstrual cycles. This demonstrated a strong potential for treatment with vitamin D and calcium to normalize cycles in women with PCOS and low levels of vitamin D.

\section{Effect of Genetic Variants of Vitamin D Metabolizing Enzymes and Proteins on PCOS Clinical Features}

In women, VDR mRNA has been shown to be expressed in the ovaries [9], the pituitary gland [40], as well as in human endometrium [41]. Thus, indicating a role of vit D in steroidogenesis of sex hormones [18] Mahmoudi found an association of VDR Apa-I polymorphism with PCOS susceptibility in a cohort including 162 PCOS and 162 control women from Tehran. Moreover, in a study conducted in Iran, there was an association of VDR Taq-I with elevated serum levels of LH as well as an association between decreased levels of SHBG and VDR Bsm-I (Mahmoudi 2009). Furthermore, several polymorphisms in the VDR gene, such as Cdx2, Taq1, Bsm1, Apa1, and Fok1, were reported to play an important role on insulin secretion and sensitivity in PCOS women [3].

In addition, the Apa1 polymorphism was reported to confer a reduced risk of vitamin D deficiency [3]. In another study, they investigated the association pattern of four VDR polymorphisms (Cdx2, Fok1, Apa1 and Taq1) with PCOS among Indian women. They found significant difference in the genotype and allele frequency distributions of the Cdx2 polymorphism between the PCOS and control women. Especially, a significantly higher frequency of the heterozygous GA genotype as well as the A allele of Cdx2 polymorphism was observed in controls when compared to patients $(\mathrm{P}<0.001)$. This reflects a protective effect of this single nucleotide polymorphism (SNP) against PCOS phenotype even after the arrangement of the covariates of age and body mass index. Moreover, they examined the correlations between VDR genotypes and some of the PCOS specific clinical/ biochemical traits and found that the Cdx2 genotypes were significantly associated with testosterone levels and the Fok1 polymorphism showed a significant association with the presence of infertility.

Furthermore, the two haplotypes composed of four polymorphisms, ACCA and ACTA, were also found to be significantly associated with PCOS Dasgupta, Dutta et al. (2015). In another cohort study on Austrian women with PCOS, the VDR Cdx2 polymorphism was found to be correlated with lower insulin resistance, and the Apa1 polymorphism was associated with lower testosterone levels [23]. However, other studies did not find significant differences in the VDR gene polymorphism frequencies between women with PCOS and normal controls [42]. On the other hand, it was reported that the VDR 1a promoter polymorphisms were not associated with the risk of PCOS but were associated with serum 25(OH)D levels in a cohort of Taiwanese Asians PCOS women [16]. In addition, in a study conducted by Ming et al, it was reported that significantly lower serum 25(OH)D levels were observed in subjects carrying the heterozygous 1521CG/1012GA haplotype of the VDR 1a promoter polymorphisms in both PCOS and control women [43].

However, metformin treatment was only effective to increase serum 25(OH)D levels in women with PCOS carrying the homozygous 1521G/1012A haplotype [16]. Despite the occurrence of several polymorphisms in VDR gene in PCOS, the results obtained from both individual research and metaanalysis in PCOS patients did not agree. As a result, the role of these variants of the VDR gene in the pathogenesis of insulin resistance and PCOS remains debatable [43].

\section{Effect of Vitamin D on Insulin Resistance}

IR is mediated through proinflamatory actions of cytokines and oxidative stress, which impairs glucose uptake and reduces insulin secretion from pancreatic $\beta$ cells [41]. Oxidative stress impairs glucose uptake in muscle and adipose tissue and reduces insulin secretion from pancreatic $\beta$ cells (Takeda et al., 2005). Several studies reported that Vitamin D reduces proinflammatory cytokines such as interferon gamma (IFN gamma) and TNF-alpha and increases the levels of anti-inflammatory cytokines such as TGF and IL-4.

Moreover, several studies proved that active Vitamin D induces several antioxidant enzymes including thioredoxin reductase [44] superoxide dismutase [13] glucose- 6 phosphate dehydrogenase [10] thus assuming Vit D can delay/contribute to prevent/treat PCOs, which needs further investigations. Vitamin D may also mediate insulin sensitivity by improving calcium status, increasing local production of $250 \mathrm{HD}$, which leads to transcriptional regulation of specific genes or suppressing serum levels of PTH [41]. Moreover, it has been proposed that obesity may have a confounding role in the relationship between 250HD and insulin resistance in women with PCOS [45].

\section{Conclusion}

Large Randomized Controlled Trials (RCTs) are highly recommended to endorse the association of calcitriol levels with metabolic and endocrine parameters in PCOS women. This might develop a promising recommendation for vitamin D supplementation to improve fertility and metabolic disturbances.

\section{Funding}

This research did not receive any specific grant from any funding agency in the public, commercial or not-for-profit sector.

\section{Duality of Interest}

The authors declare no duality of interest in the submitted review that could be perceived as prejudicing the impartiality of the research reported.

\section{Contribution Statement}

The four authors were involved in data collection, drafting, composing and the final approval of the version to be published. 


\section{Acknowledgement}

The authors of the present review would like to thank Heliopolis University for Sustainable Development and The German University in Cairo for their continuous encouragement and support to research activities.

\section{References}

1. Du, GS Daftary, SI Lalwani, HS Taylor (2005) Direct Regulation of HOXA10 by 1,25-(OH)2D3 in Human Myelomonocytic Cells and Human Endometrial Stromal Cells. Mol. Endocrinol 19(9): 2222-2233.

2. Hsieh, PW Jurutka, MA Galligan, CM Terpening, CA Haussler, et al. (1991) Human vitamin D receptor is selectively phosphorylated by protein kinase $\mathrm{C}$ on serine 51 , a residue crucial to its trans-activation function. Proc. Natl. Acad. Sci. U.S.A 88(20): 9315-9319.

3. Al-Daghri, KM Alkharfy, N Khan, AK Mohammed, B Vinodson et al (2014) Association of VDR-gene variants with factors related to the metabolic syndrome, type 2 diabetes and vitamin D deficiency. Gene 542(2): 129-133.

4. Whitfield, JC Hsieh, PW Jurutka, SH Selznick, CA Haussler, et al. (1995) Genomic actions of 1, 25-dihydroxyvitamin D3. J Nutr 125(6): 1690S-1694S.

5. Panidis, C Balaris, D Farmakiotis, D Rousso, A Kourtis, et al. (2005) Serum parathyroid hormone concentrations are increased in women with polycystic ovary syndrome. Clin. Chem 51(9): 1691-1697.

6. Adorini, L (2005) Regulation of immune responses by vitamin D receptor ligands. Vitamin D. D. Feldman, J. W. Pike and F. H. Glorieux. Elsevier/Academic Press, New York.

7. Norman, A, M Mizwicki, D Norman (2004) Steroid-hormone rapid actions, membrane receptors and a conformational ensemble model. Nat Rev Drug Discov 3(1): 27-41.

8. Huhtakangas, CJ Olivera, JE Bishop, LP Zanello, AW Norman (2004) The vitamin D receptor is present in caveolae-enriched plasma membranes and binds 1a,25(OH)2-vitamin D3 in vivo and in vitro. Mol Endo 18(11): 2660-2671.

9. Agic, C Altgassen, F Noack, Wolfler, K Diedrich, et al. (2007) Relative expression of 1,25-dihydroxyvitamin D3receptor, vitamin D 1alphahydroxylase, vitamin D 24-hydroxylase, and vitamin D 25-hydroxylase in endometriosis and gynecologic cancers. Reprod Sci 14(5): 486-497.

10. Zhang,JBao,SV Nicosia, HWang, SAEnkemann, et.al.(2005) Suppression of death receptor-mediated apoptosis by 1,25-dihydroxyvitamin D3 revealed by microarray analysis. J. Biol. Chem 280(42): 35458-35468.

11. Stephanou, A, R Ross, S Handwerger (1994) Regulation of human placental lactogen expression by 1,25-dihydroxyvitamin D3. Endocrinology 135: 2651-2656.

12. Heaney, RP, MS Dowell, CA Hale, A Bendich (2003) Calcium absorption varies within the reference range for serum 25 -hydroxyvitamin D. J Am Coll Nutr 22(2): 142-146.

13. Peehl, R Shinghal, L Nonn, E Seto, AV Krishnan, et. al. (2004) Molecular activity of 1,25-dihydroxyvitamin D3 in primary cultures of human prostatic epithelial cells revealed by cDNA microarray analysis. J Steroid Biochem Mol Biol 92(3): 131-141.

14. Swami, N Raghavachari, UR Muller, YP Bao, D Feldman (2003) Vitamin D growth inhibition of breast cancer cells: gene expression patterns assessed by cDNA microarray. Breast Cancer Res Treat 80(1): 49-62.

15. Dasgupta, J Dutta, S Annameneni, N Kudugunti, B Mohan Reddy (2015) Association of vitamin D receptor gene polymorphisms with polycystic ovary syndrome among Indian women. Indian J Med Res 142(3): 276285.
16. Lin, SJ Tsai, PY Chou, MF Huang, HS Sun, et al. (2012) Vitamin D receptor 1a promotor $-1521 \mathrm{G} / \mathrm{C}$ and $-1012 \mathrm{~A} / \mathrm{G}$ polymorphisms in polycystic ovary syndrome. Taiwan J Obstet Gynecol 51: 565-571.

17. Barrera, E Avila, G Hernández, A Halhali, B Biruete, et al. (2007) Estradiol and progesterone synthesis in human placenta is stimulated by calcitriol. Journal of Steroid Biochemistry and Molecular Biology 103(3-5): 529-532.

18. Parikh, Varadinova, P Suwandhi, T Araki, Z Rosenwaks, et al. (2010) Vitamin D regulates steroidogenesis and insulin-like growth factor binding protein-1 (IGFBP-1) production in human ovarian cells. Hormone and Metabolic Research 42: 754-757.

19. Vuolo, CD, A Faggiano, A Colao (2012) Vitamin D and cancer. Front. Endocrinol 3: 58.

20. Holick, MF (2007) Vitamin D deficiency. N Engl J Med 357(3): 266-281.

21. Kinuta, H Tanaka, H Moriwaki, K Aya, S Kato, et al. (2000) Vitamin D is an important factor in estrogen biosynthesis of both female and male gonads. Endocrinology 141(4): 1317-1324.

22. Bikle, DD (2004) Vitamin D regulated keratinocyte differentiation. J Cell Biochem 92(3): 436-444.

23. Wehr, E, TR Pieber, B Obermayer-Pietsch (2011) Effect of vitamin D3 treatment on glucose metabolism and menstrual frequency in polycystic ovary syndrome women: a pilot study. J Endocrinol Invest 34(10): 757-763.

24. Nissou, A Guttin, C Zenga, F Berger, JP Issartel, et al. (2014) Additional clues for a protective role of vitamin $\mathrm{D}$ in neurodegenerative diseases: 1,25-dihydroxyvitamin D3 triggers an anti-inflammatory response in brain pericytes. J Alzheimers Dis 42(3): 789-799.

25. Ricardo, LB (2011) VDR activation of intracellular signaling pathways in skeletal muscle. Mol Cell Endocrinol 347(1-2): 11-16.

26. Bagot, PJ Troy, HS Taylor (2000) Alteration of maternal Hoxa10 expression by in vivo gene transfection affects implantation. Gene Ther $7(16):$ 1378-1384.

27. Ramagopalan, A Heger, AJ Berlanga, NJ Maugeri, MR Lincoln, et al. (2010) A ChIP-seq defined genome-wide map of vitamin D receptor binding: associations with disease and evolution. Genome Res 20(10): 1352-1360.

28. Norman, AW (2008) From vitamin D to hormone D: fundamentals of the vitamin D endocrine system essential for good health. Am J Clin Nutr 88(2): 491S-499S.

29. Mizwicki, MT, AW Norman (2009) The vitamin D sterol-vitamin D receptor ensemble model offers unique insights into both genomic and rapid-response signaling. Sci Signal 2(75): re4.

30. Bouillon, R, H Bischoff-Ferrari, W Willett (2008) Vitamin D and health: perspectives from mice and man. J Bone Miner Res 23(7): 974-979.

31. Bouillon, R, G Carmeliet, S Boonen (1997) Ageing and calcium metabolism. Baillieres Cli Endocrinol Metab 11(2): 341-365.

32. Benn, D Ajibade, A Porta, P Dhawan, M Hediger, et al. (2008) Active intestinal calcium transport in the absence of transient receptor potential vanilloid type 6 and calbindin-D9k. Endo 149(6): 3196-3205.

33. Leibbrandt, A, J Penninger (2008) RANK/RANKL: regulators of immune responses and bone physiology. Ann N Y Acad Sci 1143: 123150.

34. Wali, RK, CL Baum, MD Sitrin, TA Brasitus (1990) 1,25(OH)2 vitamin D3 stimulates membrane phosphoinositide turnover, activates protein kinase $\mathrm{C}$, and increases cytosolic calcium in rat colonic epithelium. J. Clin. Invest 85(4): 1296-1303.

35. Morelli, C Buitrago, R Boland, AR de Boland (2001) The stimulation of MAP kinase by 1,25(OH) (2)-vitamin D (3) in skeletal muscle cells is 
mediated by protein kinase $\mathrm{C}$ and calcium. Mol. Cell. Endocrinol 173(12): 41-52.

36. Halloran, BP, HF DeLuca (1980) Effect of vitamin D deficiency on fertility and reproductive capacity in the female rat. J Nutr 110(8): 1573-1580.

37. Barrera, E Avila, G Hernández, I Méndez, L González, et al. (2008) Calcitriol affects hCG gene transcription in cultured human syncytiotrophoblasts. Reproductive Biology and Endocrinology 6: 3 .

38. Tuan, CJ Moore, JW Brittingham, JJ Kirwin, RE Akins, et al. (1991) In vitro study of placental trophoblast calcium uptake using JEG-3 human choriocarcinoma cells. Journal of Cell Science 98: 333-342.

39. ThysJacobs, S, D Donovan, A Papadopoulos (1999) Vitamin D and calcium dysregulation in the polycystic ovarian syndrome. Steroids 64 : 430-435.

40. Perez-Fernandez, M Alonso, C Segura, I Munoz, T Garcia-Caballero, et al. (1997) Vitamin D receptor gene expression in human pituitary gland. Life Sciences 60(1): 35-42.
41. Teegarden, D, SS Donkin (2009) Vitamin D: emerging new roles in insulin sensitivity. Nutr Res Rev 22(1): 82-92.

42. Moini, N Shirzad, M Ahmadzadeh, R Hosseini, L Hosseini, et al. (2015) Comparison of 25-hydroxyvitamin D and calcium levels between polycystic ovarian syndrome and normal women. Int J Fertil Steril 9: $1-8$.

43. Ming-Wei, L, W Meng-Hsing (2015) The role of vitamin D in polycystic ovary syndrome. Indian J Med Res 142(3): 238-240.

44. Greenlee, MB Hill-Harmon, T Murray, M Thun (2001) Cancer statistics, 2001. CA Cancer J Clin 51(1): 15-36.

45. Mahmoudi, T (2009) Genetic variation in the vitamin D receptor and polycystic ovary syndrome risk. Fertility and Sterility 92(4): 13811383..

\section{Your next submission with Juniper Publishers will reach you the below assets}

- Quality Editorial service

- Swift Peer Review

- Reprints availability

- E-prints Service

- Manuscript Podcast for convenient understanding

- Global attainment for your research

- Manuscript accessibility in different formats

( Pdf, E-pub, Full Text, Audio)

- Unceasing customer service

Track the below URL for one-step submission https://juniperpublishers.com/online-submission.php 